\title{
The Case for Electron Re-Acceleration at Galaxy Cluster Shocks
}

On the largest scales, the Universe consists of voids and filaments making up the cosmic web. Galaxy clusters are located at the knots in this web, at the intersection of filaments. Clusters grow through accretion from these large-scale filaments and by mergers with other clusters and groups. In a growing number of galaxy clusters, elongated Mpc-size radio sources have been found [1, 2]. Also known as radio relics, these regions of diffuse radio emission are thought to trace relativistic electrons in the intracluster plasma accelerated by low-Mach number shocks generated by cluster-cluster merger events [3]. A long-standing problem is how low-Mach number shocks can accelerate electrons so efficiently to explain the observed radio relics. Here we report the discovery of a direct connection between a radio relic and a radio galaxy in the merging galaxy cluster Abell 3411-3412 by combining radio, X-ray, and optical observations. This discovery indicates that fossil relativistic electrons from active galactic nuclei are re-accelerated at cluster shocks. It also implies that radio galaxies play an important role in governing the non-thermal component of the intracluster medium in merging clusters.

Cluster mergers are the most energetic events in the Universe after the Big Bang, releasing energies up to $\sim 10^{64} \mathrm{ergs}$ on Gyr timescales. Most of the gravitational energy released during cluster merger events is converted into thermal energy via low-Mach number shocks $(\mathcal{M} \lesssim 3)$ and turbulence in the intracluster medium (ICM) [4]. A small fraction $(\lesssim 1 \%)$ of the energy dissipated at shocks could be channeled into the acceleration of cosmic rays (CR). In the presence of magnetic fields, CR electrons would then emit synchrotron radiation which can be observed with radio telescopes. The origin of the large-scale magnetic fields, and the nature of particle acceleration processes that operate in these dilute cosmic plasmas, are still open questions.

The ICM has a high thermal-to-magnetic pressure ratio, or $\beta$, and electron acceleration by lowMach number collisionless shocks in such high- $\beta$ plasmas is poorly understood, as analytical calculations cannot properly capture the non-linear behavior of this process [5]. Radio relics, elongated sources that trace the CR at ICM shocks, provide us with rare opportunities to probe this process. While there is substantial evidence that relics trace CR electrons at shocks [6,7], previous work has found that the acceleration efficiency should be very low at these shocks, if these synchrotron emitting electrons are accelerated from the thermal pool of the ICM via the diffusive shock acceleration (DSA) mechanism [5]. This low efficiency is hard to reconcile with the observed brightness of some radio relics, suggesting a high acceleration efficiency [8-10]. In addition, some relics have regions with rather flat radio spectra $\left(\alpha \approx-0.7 ; F_{\nu} \propto \nu^{\alpha}\right.$, where $\alpha$ is the spectral index), but the corresponding shock Mach numbers measured via X-ray observations are low [11,12]. This contradicts with the prediction from DSA [13]. This long-standing problem has 
so far remained unsolved. Furthermore, large merger shocks have also been found without corresponding radio relics [14], indicating that our understanding of particle acceleration by low-Mach number shocks is still incomplete.

Recently, new insights into the acceleration by low-Mach number shocks have been obtained by particle-in-cell (PIC) simulations [15]. These PIC simulations show that acceleration from the thermal pool could be possible. However, for some relics an unrealistic fraction of the shock energy needs to be transferred into the non-thermal electron population to explain their radio brightness $[12,16,17]$ and the PIC simulations do not solve this problem. A solution to explain the apparent very efficient acceleration, is to invoke the presence of a population of fossil relativistic electrons [18], with Lorentz factors $\gamma \gtrsim 10^{2}$. The synchrotron lifetime of relativistic electrons in the ICM is relatively short $\left(\sim 10^{8} \mathrm{yrs}\right)$. Once these electrons have lost most of their energy, they do not radiate within the observable radio band and they thus become invisible to radio telescopes. It has been suggested that these fossil electrons, which have Gyr lifetimes, can be efficiently reaccelerated at shocks and are therefore able to create bright radio relics $[9,19]$. Obvious candidates for these fossil electrons are the (old) lobes and tails of radio galaxies [7, 20, 21]. This fossil radio plasma can occupy a significant volume of the ICM due to turbulent diffusion, aided by the random galaxy motions throughout the ICM and the ICM motion itself. Indeed, observational evidence has been found that provides support for this model. The complex morphologies of some radio relics resemble those of disturbed tailed radio galaxies that are often found in merging galaxy clusters [7, 20]. The relics in the Bullet Cluster and PLCKG287.0+32.9 are two prime examples [7, 22]. However, for the cluster PLCKG287.0+32.9, no redshift for the putative radio galaxy connected to the relic could be obtained, since the proposed core of the radio galaxy did not have an optical counterpart [20]. Similarly, no direct link between the relic in the Bullet cluster and a tailed radio galaxy could be established [7]. The relic in the Coma cluster provides another interesting case. Here a confirmed cluster radio galaxy seems to be connected to a relic [21]. Adiabatic compression of fossil plasma by a shock was proposed as a model for its origin [23,24]. While the electrons do gain energy by the adiabatic compression, this model does not invoke any Fermi-type re-acceleration processes.

Here we present optical, radio, and X-ray observations of the merging galaxy cluster Abell 34113412 located at $z=0.162$. This cluster contains a Mpc-size radio relic with an irregular shape $[25,26]$. Chandra observations, totaling $211 \mathrm{ks}$, reveal a cluster with a cometary morphology undergoing a major merger event, with the compact core of one of the subclusters being the "head" of the comet (Fig. 1). A dynamical analysis based on Keck spectra of 174 cluster members and Subaru imaging, indicates that this is an approximately 1:1 mass ratio merger viewed $\sim 1$ Gyr after core passage, with the merger axis being located close to the plane of the sky. The two subclusters are both very massive, with individual masses of $\sim 10^{15} \mathrm{M}_{\odot}$. The core of the cluster coming in from the south (and currently observed in the north) was compact enough to survive the collision with the other cluster up to the present time, while the gas core of the subcluster that came in from the north was largely disrupted during the core passage.

The Giant Metrewave Radio Telescope (GMRT) radio images at 610 and $325 \mathrm{MHz}$ show the 
complex, large radio relic located in the southern outskirts of the merging cluster (Fig. 2). Most interestingly, a close inspection of the northeastern component of the radio relic reveals an elongated narrow extension that leads from the relic towards a galaxy (source A, Figs. 2 and 3a). This galaxy is a spectroscopically confirmed cluster member $(z=0.164)$. A high-resolution $3 \mathrm{GHz}$ Very Large Array (VLA) image shows an active galactic nucleus (AGN; radio core) at the galaxy's center. From the core, a narrow tail "feeds" into the radio relic located $\approx 90 \mathrm{kpc}$ to its south. This reveals a direct connection between the relativistic plasma of an AGN and that of a radio relic. The probability of a chance projection of a tailed radio galaxy with this relic is 0.004 .

Our radio spectral index measurements, between 0.325 and $3.0 \mathrm{GHz}$ show that the radio spectrum steepens away with distance from the compact nucleus from $\alpha=-0.5 \pm 0.1$ to $\alpha=-1.3 \pm 0.1$ ( $1 \sigma$ errors), as is expected for synchrotron and Inverse Compton losses (Fig. 3b). Remarkably, where the radio plasma from the AGN connects to the radio relic, the spectrum flattens back to $\alpha=-0.9 \pm 0.1$. This flattening is strong evidence for the re-acceleration of electrons from the radio tail at a shock. Moreover, we find evidence for spectral steepening across the relic in the direction towards the cluster center. These gradients have routinely been found for other relics and they are explained by electron energy losses in the downstream region of an outwards traveling shock [27]. We also find the outer edge of the relic is polarized, with a maximum polarization fraction of $40 \%$. The emission weighted average polarization fraction is $13 \%$ (Fig. 3c). The relatively modest spectral flattening at the shock suggests that the shock cannot be very strong. Furthermore, the existence of a downstream spectral index gradient (see Fig. 3b) suggests that the relic cannot be solely caused by the adiabatic compression of a lobe of fossil radio plasma.

To search for the shock, we extracted a $0.5-2 \mathrm{keV}$ Chandra surface brightness profile in a sector containing the relic and fitted the profile by assuming an underlying broken power-law density model. At large radii, the density profiles of galaxy clusters are typically described by single power-laws. However, the profile across the NE part of the relic steepens swiftly near the relic's outer edge and shows a significant departure from a single power-law, indicating a deviation from hydrostatic equilibrium as expected for a shock (Fig. 4). From the profile we find that the shock must be rather weak, with $\mathcal{M} \lesssim 1.7$. This upper limit should be considered an approximate estimate due to the idealized assumptions about the shock geometry and projection effects.

Our observations of (1) a direct connection between a radio galaxy and the relic, (2) spectral flattening at the location where the radio tail meets the relic, (3) the presence of an X-ray surface brightness discontinuity at the relic's outer edge, and (4) a high polarization fraction at the location of flattest spectral indices provide the best evidence to date that radio galaxies in clusters provide seed electrons that can be re-accelerated and revived by merger shocks. Re-acceleration (and also adiabatic compression) alleviates the problem of the low-acceleration efficiency from the thermal pool. In addition, it provides a natural explanation why some cluster merger shocks have no corresponding radio relics. Those cases may lack a sufficient supply of fossil radio plasma. Finally, re-acceleration also solves the problem of the relatively flat spectral indices observed from relics, which for DSA, requires shocks that have higher Mach numbers than observed in X-rays $[11,12]$.

Different re-acceleration models have been proposed. In one of these models, fossil relativis- 
tic electrons are re-accelerated by a DSA-like process $[18,28]$ in combination with adiabatic compression. In another model, the re-acceleration happens in the shock downstream region by magneto-hydrodynamical turbulence [28]. Future work is needed to determine which of these reacceleration mechanisms operates at radio relics [29], and determine whether re-acceleration is required to explain all relics. We evaluate one of these re-acceleration models in the Supplementary Information to investigate what Mach number is required to flatten the spectral index by the observed amount and examine the relatively uniform spectral index along the length of the shock front.

The Abell 3411-3412 cluster contains at least two additional radio galaxies about $2^{\prime}$ to the south and $2^{\prime}$ to southwest of the one described above (sources B and C; Fig. 2). One of the radio galaxies is embedded within the relic emission, for the other, a tail of steep spectrum radio emission extends towards the relic. We argue that it is therefore likely that the other components of the complex relic in Abell 3411-3412 also trace revived fossil plasma, either by the process of adiabatic compression or by re-acceleration. Re-acceleration should also operate in other clusters because more examples of radio galaxies near relics have been found (i.e., in the Coma Cluster [21]). Our findings imply that PIC simulations and laboratory laser experiments [30] for collisionless shocks should include a relativistic fossil electron distribution in the upstream plasma. This study also indicates that to understand the non-thermal component of the ICM, the presence and distribution of radio galaxies needs to be taken into account, in addition to particle acceleration at shocks. Observations at low radio frequencies, in particular with LOFAR, will be key to unveiling the connections between relics and AGN, because low-frequency observations are sensitive to older, fossil radio plasma.

\section{References}

[1] Feretti, L., Giovannini, G., Govoni, F., Murgia, M. Clusters of galaxies: observational properties of the diffuse radio emission. Astron. Astrophys. Rev. 20, 54 (2012).

[2] Brunetti, G., Jones, T. W. Cosmic Rays in Galaxy Clusters and Their Nonthermal Emission. Int. J. Mod. Phys. D 23, 1430007-98 (2014).

[3] Ensslin, T. A., Biermann, P. L., Klein, U., Kohle, S. Cluster radio relics as a tracer of shock waves of the large-scale structure formation. Astron. Astrophys. 332, 395-409 (1998).

[4] Ryu, D., Kang, H., Hallman, E., Jones T. W. Cosmological Shock Waves and Their Role in the Large-Scale Structure of the Universe Astrophys. J. 593, 599-610 (2003).

[5] Kang, H., Ryu, D. Cosmic ray spectrum from diffusive shock acceleration. Astrophys. Space Sci. 336, 263-268 (2011).

[6] Macario, G., Markevitch, M., Giacintucci, S., Brunetti, G., Venturi, T., Murray, S. S. A Shock Front in the Merging Galaxy Cluster A754: X-ray and Radio Observations. Astrophys. J. 728, 82 (2011). 
[7] Shimwell, T. W., Markevitch, M., Brown, S., Feretti, L., Gaensler, B. M., Johnston-Hollitt, M., Lage, C., Srinivasan, R. Another shock for the Bullet cluster, and the source of seed electrons for radio relics. Mon. Not. R. Astron. Soc. 449, 1486-1494 (2015).

[8] Kang, H. and Ryu, D. and Jones, T. W. Diffusive Shock Acceleration Simulations of Radio Relics. Astrophys. J. 756, 97 (2012).

[9] Pinzke, A. and Oh, S. P. and Pfrommer, C. Giant radio relics in galaxy clusters: reacceleration of fossil relativistic electrons? Mon. Not. R. Astron. Soc. 435, 1061-1082 (2013).

[10] Vazza, F. and Brüggen, M. Do radio relics challenge diffusive shock acceleration? Mon. Not. R. Astron. Soc. 437, 2291-2296 (2014).

[11] Itahana, M., Takizawa, M., Akamatsu, H., Ohashi, T., Ishisaki, Y., Kawahara, H., van Weeren, R. J. Suzaku observations of the galaxy cluster 1RXS J0603.3+4214: Implications of particle acceleration processes in the "Toothbrush" radio relic. Publ. Astron. Soc. Jpn. 67, 113 (2015).

[12] van Weeren, R. J., Brunetti, G., Brüggen, M., Andrade-Santos, F., Ogrean, G. A., Williams, W. L., Röttgering, H. J. A., Dawson, W. A., Forman, W. R., de Gasperin, F., Hardcastle, M. J., Jones, C., Miley, G. K., Rafferty, D. A., Rudnick, L., Sabater, J., Sarazin, C. L., Shimwell, T. W., Bonafede, A., Best, P. N., Bîrzan, L., Cassano, R., Chyży, K. T., Croston, J. H., Dijkema, T. J., Enßlin, T., Ferrari, C., Heald, G., Hoeft, M., Horellou, C., Jarvis, M. J., Kraft, R. P., Mevius, M., Intema, H. T., Murray, S. S., Orrú, E., Pizzo, R., Sridhar, S. S., Simionescu, A., Stroe, A., van der Tol, S., White, G. J. LOFAR, VLA, and Chandra Observations of the Toothbrush Galaxy Cluster. Astrophys. J. 818, 204 (2016).

[13] Blandford, R. and Eichler, D. Particle acceleration at astrophysical shocks: A theory of cosmic ray origin. Phys. Rep. 154, 1-75 (1987).

[14] Russell, H. R., van Weeren, R. J., Edge, A. C., McNamara, B. R., Sanders, J. S., Fabian, A. C., Baum, S. A., Canning, R. E. A., Donahue, M., O’Dea, C. P. A merger mystery: no extended radio emission in the merging cluster Abell 2146. Mon. Not. R. Astron. Soc. 417, 1-5 (2011).

[15] Guo, X. and Sironi, L. and Narayan, R. Non-thermal Electron Acceleration in Low Mach Number Collisionless Shocks. II. Firehose-mediated Fermi Acceleration and its Dependence on Pre-shock Conditions. Astrophys. J. 797, 47 (2014).

[16] Botteon, A., Gastaldello, F., Brunetti, G., Dallacasa, D. A shock at the radio relic position in Abell 115. Mon. Not. R. Astron. Soc. 460, 84-88 (2016).

[17] Eckert, D., Jauzac, M., Vazza, F., Owers, M. S., Kneib, J.-P., Tchernin, C., Intema, H., Knowles, K. A shock front at the radio relic of Abell 2744. Mon. Not. R. Astron. Soc. 461, 1302-1307 (2016).

[18] Markevitch, M., Govoni, F., Brunetti, G., Jerius, D. Bow Shock and Radio Halo in the Merging Cluster A520. Astrophys. J. 627, 733-738 (2005). 
[19] Kang, H. and Ryu, D. Curved Radio Spectra of Weak Cluster Shocks. Astrophys. J. 809, 186 (2015).

[20] Bonafede, A., Intema, H. T., Brüggen, M., Girardi, M., Nonino, M., Kantharia, N., van Weeren, R. J., Röttgering, H. J. A. Evidence for Particle Re-acceleration in the Radio Relic in the Galaxy Cluster PLCKG287.0+32.9. Astrophys. J. 785, 1 (2014).

[21] Giovannini, G., Feretti, L., Stanghellini, C. The Coma cluster radio source $1253+275$, revisited. Astron. Astrophys. 252, 528-537 (1991).

[22] Bagchi, J., Sirothia, S. K., Werner, N., Pandge, M. B., Kantharia, N. G., Ishwara-Chandra, C. H., Gopal-Krishna, Paul, S., Joshi, S. Discovery of the First Giant Double Radio Relic in a Galaxy Cluster Found in the Planck Sunyaev-Zel'dovich Cluster Survey: PLCK G287.0+32.9. Astrophys. J. 736, 8 (2011).

[23] Enßlin, T. A. \& Gopal-Krishna, Reviving fossil radio plasma in clusters of galaxies by adiabatic compression in environmental shock waves. Astron. Astrophys., 366, 26-34 (2001).

[24] Enßlin, T. A. \& Brüggen, M. On the formation of cluster radio relics. Mon. Not. R. Astron. Soc., 331, 1011-1019 (2002).

[25] van Weeren, R. J., Fogarty, K., Jones, C., Forman, W. R., Clarke, T. E., Brüggen, M., Kraft, R. P., Lal, D. V., Murray, S. S., Röttgering, H. J. A. Complex Diffuse Radio Emission in the Merging Planck ESZ Cluster A3411. Astrophys. J. 769, 101 (2013).

[26] Giovannini, G. and Vacca, V. and Girardi, M. and Feretti, L. and Govoni, F. and Murgia, M. The nature of the giant diffuse non-thermal source in the A3411-A3412 complex. Mon. Not. R. Astron. Soc. 435, 518-523 (2013).

[27] van Weeren, R. J., Röttgering, H. J. A., Brüggen, M., Hoeft, M. Particle Acceleration on Megaparsec Scales in a Merging Galaxy Cluster. Science 330, 347 (2010).

[28] Fujita, Y., Takizawa, M., Yamazaki, R., Akamatsu, H., Ohno, H. Turbulent Cosmic-Ray Reacceleration at Radio Relics and Halos in Clusters of Galaxies. Astrophys. J. 815, 116 (2015).

[29] Vazza, F., Eckert, D., Brüggen, M., Huber, B. Electron and proton acceleration efficiency by merger shocks in galaxy clusters. Astrophys. J. 451, 2198-2211 (2015).

[30] Stockem, A. and Fiuza, F. and Bret, A. and Fonseca, R. A. and Silva, L. O. Exploring the nature of collisionless shocks under laboratory conditions. Nature Scientific Reports 3934 (2014).

Correspondence Correspondence and requests for materials should be addressed to R.J.W. (email: rvanweeren@cfa.harvard.edu). 
Acknowledgements Support for this work was provided by the National Aeronautics and Space Administration through Chandra Award Number GO5-16133X issued by the Chandra X-ray Observatory Center, which is operated by the Smithsonian Astrophysical Observatory for and on behalf of the National Aeronautics Space Administration under contract NAS8-03060. We thank the staff of the GMRT who have made these observations possible. The GMRT is run by the National Centre for Radio Astrophysics of the Tata Institute of Fundamental Research. The National Radio Astronomy Observatory is a facility of the National Science Foundation operated under cooperative agreement by Associated Universities, Inc. Based on observations obtained at the Southern Astrophysical Research (SOAR) telescope, which is a joint project of the Ministério da Ciência, Tecnologia, e Inovação (MCTI) da República Federativa do Brasil, the U.S. National Optical Astronomy Observatory (NOAO), the University of North Carolina at Chapel Hill (UNC), and Michigan State University (MSU). Based on data collected at Subaru Telescope, which is operated by the National Astronomical Observatory of Japan. Part of this work performed under the auspices of the U.S. DOE by LLNL under Contract DE-AC52-07NA27344. Some of the data presented herein were obtained at the W.M. Keck Observatory, which is operated as a scientific partnership among the California Institute of Technology, the University of California and the National Aeronautics and Space Administration. The Observatory was made possible by the generous financial support of the W.M. Keck Foundation. The Isaac Newton Telescope is operated on the island of La Palma by the Isaac Newton Group in the Spanish Observatorio del Roque de los Muchachos of the Instituto de Astrofísica de Canarias. R.J.W. is supported by a Clay Fellowship awarded by the Harvard-Smithsonian Center for Astrophysics. V.M.P. acknowledges support for this work from grant PHY 14-30152; Physics Frontier Center/JINA Center for the Evolution of the Elements (JINA-CEE), awarded by the US National Science Foundation. D.R. was supported by the National Research Foundation of Korea through grant 2016R1A5A1013277. H.K. was supported by the National Research Foundation of Korea through grant 2014R1A1A2057940. R.M.S. acknowledges CAPES (PROEX), CNPq, PRPG/USP, FAPESP and INCT-A funding. M.J.J. acknowledges the support from NRF of Korea to CGER. D.S. acknowledges financial support from the Netherlands Organisation for Scientific research (NWO) through a Veni fellowship. G.A.O. is supported by NASA through Hubble Fellowship grant \#HST-HF2-51345.001-A, awarded by the Space Telescope Science Institute, which is operated by the Association of Universities for Research in Astronomy, Inc., for NASA, under contract NAS5-26555.

Author Contributions R.J.W. coordinated the research, wrote the manuscript, reduced the VLA data, and led the Chandra observing proposal. F.A.-S., K.F., and G.A.O. performed the Chandra data reduction and worked on the X-ray surface brightness profile fitting. H.K. and D.R. carried out the re-acceleration modeling. M.B., W.R.F., and C.J. helped with the interpretation of the radio and X-ray results and provided extensive feedback on the manuscript. C.J. led the GMRT observing proposal. D.V.L. obtained the GMRT observations and carried out the GMRT data reduction. V.M.P. and R.M.A. obtained the SOAR observations and performed the corresponding data reduction. D.S. and A.S. provided the INT observations and reduced the data. W.A.D. carried out the dynamical modeling of the merger event. W.A.D, N.G. and M.J.J. obtained the Keck and Subaru observations and reduced the data. D.W. helped with the interpretation of the dynamical modeling and led the Keck and Subaru observing proposals. R.P.K. assisted with the writing of the Chandra observing proposal.

\section{Author Information}

Reinout J. van Weeren ${ }^{1}$, Felipe Andrade-Santos ${ }^{1}$, William A. Dawson ${ }^{2}$, Nathan Golovich ${ }^{3}$, Dharam V. Lal ${ }^{4}$, Hyesung Kang ${ }^{5}$, Dongsu Ryu ${ }^{6,7}$, Marcus Brüggen ${ }^{8}$, Georgiana A. Ogrean ${ }^{9}$, William R. Forman ${ }^{1}$, Christine Jones ${ }^{1}$, Vinicius M. Placco ${ }^{10}$, Rafael M. Santucci ${ }^{11}$, David Wittman ${ }^{3,12}$, M. James Jee ${ }^{13}$, Ralph P. Kraft ${ }^{1}$, 
David Sobral $^{14,15}$, Andra Stroe ${ }^{16} \&$ Kevin Fogarty ${ }^{17}$

${ }^{1}$ Harvard-Smithsonian Center for Astrophysics, 60 Garden Street, Cambridge, MA 02138, USA

${ }^{2}$ Lawrence Livermore National Lab, 7000 East Avenue, Livermore, CA 94550, USA

${ }^{3}$ University of California, One Shields Avenue, Davis, CA 95616, USA

${ }^{4}$ National Centre for Radio Astrophysics, TIFR, Pune University Campus, Post Bag 3, Pune 411007, India

${ }^{5}$ Department of Earth Sciences, Pusan National University, Busan 46241, Korea

${ }^{6}$ Department of Physics, UNIST, Ulsan 44919, Korea

${ }^{7}$ Korea Astronomy and Space Science Institute, Daejeon 34055, Korea

${ }^{8}$ Hamburger Sternwarte, Hamburg University, Gojenbergsweg 112, 21029 Hamburg, Germany

${ }^{9}$ Kavli Institute for Particle Astrophysics and Cosmology, Stanford University, 452 Lomita Mall, Stanford, CA 94305-4085, USA

${ }^{10}$ Department of Physics and JINA Center for the Evolution of the Elements, University of Notre Dame, Notre Dame, IN 46556, USA

${ }^{11}$ Departamento de Astronomia - Instituto de Astronomia, Geofísica e Ciências Atmosféricas, Universidade de São Paulo, São Paulo, SP 05508-900, Brazil

${ }^{12}$ Instituto de Astrofísica e Ciências do Espaço, Universidade de Lisboa, Lisbon 1749-016, Portugal

${ }^{13}$ Department of Astronomy and Center for Galaxy Evolution Research, Yonsei University, 50 Yonsei-ro, Seoul 03722, Korea

${ }^{14}$ Department of Physics, Lancaster University, Lancaster LA1 4YB, UK

${ }^{15}$ Leiden Observatory, Leiden University, PO Box 9513, NL-2300 RA Leiden, the Netherlands

${ }^{16}$ European Southern Observatory, Karl-Schwarzschild-Straße 2, D-85748 Garching bei Müchen, Germany

${ }^{17}$ Department of Physics and Astronomy, The Johns Hopkins University, 3400 North Charles Street, Baltimore, MD 21218-2686, USA

Competing Interests The authors declare no competing financial interests.

\section{Figure legends}

Figure 1: Subaru gri color image of the merging cluster Abell 3411-3412. Radio emission at $610 \mathrm{MHz}$ from the GMRT is shown in red. The 0.5-2.0 keV Chandra X-ray image is shown in blue. The galaxy distribution is shown with white contours. Contour levels are drawn at $[3,4,5, \ldots]$ galaxies $\operatorname{arcmin}^{-2}$. The dashed box indicates the region shown in Fig. 3. 
Figure 2: GMRT radio images. (a) GMRT $610 \mathrm{MHz}$ image. Contours are drawn at levels of $\sqrt{[1,2,4, \ldots]} \times 4 \sigma_{\text {rms }}$, with $\sigma_{\text {rms }}$ the map noise. The beam size is indicated in the bottom left corner. The dashed box indicates the region shown in Fig. 3. Cluster radio galaxies are labeled A, B, and C. (b) GMRT $325 \mathrm{MHz}$ image. Contour levels are drawn as in the left panel. Dotted contours are drawn at $-3 \sigma_{\text {rms }}$.

Figure 3: Subaru optical, radio spectral index, and polarization maps of the of the northeast component of the radio relic in Abell 3411-3412. The beam sizes are indicated in the bottom left corner of the images. (a) Subaru gri color image. GMRT $610 \mathrm{MHz}$ contours are overlaid in yellow. Contours are drawn at levels of $\sqrt{[1,2,4, \ldots]} \times 5 \sigma_{\text {rms. }}$ (b) A spectral index map. The spectral index was determined using matched observations at frequencies of $0.325,0.61,1.5$ and $3.0 \mathrm{GHz}$. Contours are from the GMRT $325 \mathrm{MHz}$ image and are drawn at levels of $[1,2,4, \ldots] \times 6 \sigma_{\mathrm{rms}}$. The dashed line indicates the best-fitting position of the X-ray surface brightness edge. (c) Polarization map at $3.0 \mathrm{GHz}$. Vectors display the electric field angles. The length of the vectors is proportional to the polarization fraction. A reference vector for $100 \%$ polarization is shown in the bottom left corner. The greyscale image shows the linear polarized intensity. Black contours trace the Stokes I continuum image and are drawn at $[0.03,0.12] \mathrm{mJy}_{\text {beam }}{ }^{-1}$.

Figure 4: Chandra 0.5-2.0 keV surface brightness profile across the radio relic in an elliptical sector (see the SI). Uncertainties on the data points are plotted at the $1 \sigma$ level. The blue line corresponds to the best-fitting density model. This double power-law model fits a jump (at a distance of $\approx 4^{\prime}$ ) with a density compression factor $(C)$ of $1.31_{-0.09}^{+0.10}$. The position of this jump is indicated on the spectral index image (Fig. 3). 


\section{Methods}

\section{Radio observations, data reduction, and spectral indices}

Abell 3411-3412 was observed with the GMRT on 21 Oct and 19 Nov, 2012, at 610 and $325 \mathrm{MHz}$, respectively. A total bandwidth of $32 \mathrm{MHz}$ was recorded. The on source time was $4.5 \mathrm{hrs}$ at $325 \mathrm{MHz}$ and $3.6 \mathrm{hrs}$ at $610 \mathrm{MHz}$. The initial calibration and visual removal of radio frequency interference (RFI) were carried out with AIPS (http://www.aips.nrao.edu/). The initial calibration consisted of bandpass calibration, bootstrapping of the flux-density scale, and the transfer of gains from the calibrator sources to the target field. The calibration solutions were further refined via the process of self-calibration using the CASA package [31]. For the imaging, W-projection was employed to account for the non-coplanar nature of the array $[32,33]$. For the weighting, we employed the Briggs scheme with a robust parameter of 0.0 , unless mentioned. The $610 \mathrm{MHz}$ image has a rms noise $\left(\sigma_{\mathrm{rms}}\right)$ of $35 \mu \mathrm{Jy}$ beam $^{-1}$ and a resolution of $6.1^{\prime \prime} \times 5.1^{\prime \prime}$. The $325 \mathrm{MHz}$ image has a resolution of $10.5^{\prime \prime} \times 8.3^{\prime \prime}$ and $\sigma_{\mathrm{rms}}=87 \mu \mathrm{Jy}$ beam $^{-1}$.

The cluster was also observed with the Karl G. Jansky VLA, covering the 2-4 GHz S-band in B-array on $26 \mathrm{Feb}, 2015$ and in DnC-array on 7 Jan, 2016 (project SG0455). The time on source was $1.2 \mathrm{hr}$ for both observing runs. In addition, 1-2 GHz L-band observations (project 15A-270) were obtained in BnA-array on 18 May, 2015 with a total time on source of $0.5 \mathrm{hr}$. These observations were reduced and calibrated with CASA following the process outlined in [34]. RFI was automatically removed with the AOFlagger [35]. The calibration consisted of delay, bandpass, and gain corrections. The channel dependent polarization leakage and angles were calibrated using the calibrators $3 \mathrm{C} 147$ and $3 \mathrm{C} 138$. The solutions were transferred to the target field and the solutions were further refined via the process of self-calibration. The imaging was carried out in the same way as the GMRT data. For imaging of the L-band data, we also used 1.4 GHz VLA D-array observations (project AC0696) to include emission on larger scales that are missed by the B-array observations. The reduction and calibration of the D-array observations have been described in [25]. All images were corrected for the primary beam attenuation.

For making spectral index maps, we created images at 0.325 and $3.0 \mathrm{GHz}$. To correct for the different sampling densities in the uv-plane, uniform weighting was employed. We also placed inner uv-range cuts to image only common uv-ranges. The images were then convolved to the lowest resolution, given by the $325 \mathrm{MHz}$ GMRT observations $\left(9.1^{\prime \prime} \times 6.5^{\prime \prime}\right)$. The spectral index map for the area around A (for the labeling see Fig. 2) is shown in the Supplementary Information (SI). We also computed a higher signal-to-noise spectral index map, including the 0.61 and $1.5 \mathrm{GHz}$ observations. As before, the 0.61 and $1.5 \mathrm{GHz}$ observations were imaged with uniform weighing and inner uv-range cuts. To compute the spectral index, we fitted power-law spectra through the four data points, ignoring any possible curvature. Pixels with values below $4 \sigma_{\text {rms }}$ were "blanked". The use of four frequencies decreases the noise on the derived spectral indices compared to only using the 0.325 and $3.0 \mathrm{GHz}$ images. The spectral index map and corresponding uncertainties, under the assumption that the spectra can be described by power-laws, are shown in the SI (a cutout around source A is shown in Fig. 3b). 
The S-band polarization image is shown in Fig 3c. This images has a resolution of $10^{\prime \prime} \times 8^{\prime \prime}$. No vectors are plotted for pixels where the signal-to-noise ratio of the polarized intensity is $<4$. Given the low Galactic Faraday Rotation Measure of $\approx-7 \mathrm{rad} \mathrm{m}^{-1}$, we did not correct the polarization angles for Faraday Rotation [36]. The high-resolution S-band continuum map, showing the radio core associated with the AGN of source A, is shown in the SI.

We computed the probability of a chance projection of the tailed radio galaxy with the relic attached to it. This was done by taking the ratio of the area covered by the relic and the cluster, with the cluster area given by $\pi * R_{500}^{2}$, and $R_{500} \approx 1.4 \mathrm{Mpc}$. This results in $p=0.002$. Taking the conservative approach, considering that the cluster contains two disturbed radio galaxies, the probability of a chance projection increases to $p=0.004$.

The cluster contains at least two more radio galaxies ( $\mathrm{B}$ and $\mathrm{C}$, see Fig. 2) to the south and southwest of the one described above. One of the radio galaxies (source B) is embedded within the relic emission. Source C is a distorted FR-I [37] source. The spectral index map and the $325 \mathrm{MHz}$ image (SI and Fig. 2) reveal a hint of a connection between source's C southern lobe/tail and the southernmost part of the relic. The spectral index steepens along the lobes of source $\mathrm{C}$, as expected due to synchrotron and Inverse Compton losses. Given that the relic emission around sources B and $\mathrm{C}$ is located further to south than the relic near source $\mathrm{A}$, it is seems unlikely that the same shock is responsible for reviving the fossil plasma in all these regions. This would suggest that the

ICM in the extreme southern outskirts of the cluster is disturbed by other shocks. Our Chandra observations do show the presence of additional substructure in the general area around source B and $\mathrm{C}$. However, given the low counts rates it is not possible to extract detailed information on the thermal properties of the ICM in this region.

\section{Chandra observations and data reduction.}

The cluster was observed with ACIS-I on the Chandra X-ray Observatory for a total of 8 times between 2012 and 2015. This resulted in a total exposure time of $211 \mathrm{ks}$. The data were reduced with the chav package, following the processing described in [38] and applying the CALDB 4.6.7 calibration files. This processing includes filtering of bad events by checking for periods of high background, corrections for the time dependence of the charge transfer inefficiency and gain, removal of readout artifacts, background subtraction, and exposure correction. For the background subtraction, we used standard blank sky background files. The images of the separate exposures were then combined into a single image, binning with a factor of 4 (i.e., $2^{\prime \prime}$ pixel ${ }^{-1}$ ). 


\section{Surface brightness profile fitting.}

We fit the X-ray surface brightness profile with an updated version of Proffit $[39,40]$. We model the profile using an underlying broken power-law density model

$$
n(r)= \begin{cases}C n_{0}\left(\frac{r}{r_{\text {edge }}}\right)^{a_{2}}, & r<r_{\text {edge }} \\ n_{0}\left(\frac{r}{r_{\text {edge }}}\right)^{a_{1}}, & r>r_{\text {edge }} .\end{cases}
$$

The subscripts 1 and 2 refer to the up and downstream regions, respectively. The parameter $r_{\text {edge }}$ denotes the location of the jump, $n_{0}$ is the normalization constant, $C$ the shock compression factor, $a_{1}$ and $a_{2}$ are the slopes of the power-laws. This density model is then projected along the line of sight to obtain an X-ray surface brightness profile, assuming prolate spheroidal geometry within the sector. The emissivity is taken to be proportional to the density squared.

In the case of a shock, the compression factor can be related to the shock Mach number

$$
\mathcal{M}=\left[\frac{2 C}{\gamma+1-C(\gamma-1)}\right]^{1 / 2},
$$

where $\gamma$ is the adiabatic index of the gas. We assume $\gamma=5 / 3$ for the thermal plasma.

We fit the surface brightness profile in an elliptical sector that crosses the radio relic (see the SI), using the model given in Eq. 1. The opening angles $\left(210^{\circ}-250^{\circ}\right)$ were chosen after visual inspection of the Chandra image (see the SI), which indicates a possible surface brightness edge at the relic location and extending $\sim 10^{\circ}$ further to the west. For the radial binning of photons, we require a SNR of 5 per bin. We exclude the regions affected by point sources during the fitting. The observed profile and most best fitting model is shown in Fig. 4. We find a break in the X-ray surface brightness profile at the location of the radio relic. The resulting density jump is small $C=1.31_{-0.09}^{+0.10}(\mathcal{M}=1.2)$, with a $90 \%$ confidence upper limit of $C<1.56$, indicating that the Mach number is low $\mathcal{M}<1.4$. The $90 \%$ lower confidence limit is $C>1.16$. The location of the jump is indicated in Fig. $3 b$.

We also extracted a profile in a spherical sector (see the SI). The resulting fitted compression factor is slightly lower with $C \approx 1.2$, but consistent with the previous results. In addition, we checked whether the results changed for a smaller opening angle, corresponding to the relic's visible extent in the 610 and $325 \mathrm{MHz}$ images (i.e., an opening angle of $210^{\circ}-240^{\circ}$ ). This did not result in significant changes for the values of the compression factor or discontinuity location. We also slightly varied the placement of the sector, again obtaining consistent results. Therefore we conclude that the presence of a jump does not depend on the precise sector placement and shape. For comparison, in the SI we show a model with a density jump of $C=2.3(\mathcal{M}=2.0)$ in the elliptical sector, which does not provide a good match to the data.

A small "bump" is visible in the X-ray surface brightness profile at a radial distance of $4.5^{\prime}$ 5. $0^{\prime}$ (Fig. 4). The nature of this bump is unclear. Ignoring this bump increases the best-fitting compression factor and the $90 \%$ confidence upper limit to $C<2.0(\mathcal{M}<1.7)$. 
From the X-ray surface brightness profile we conclude that there is evidence for a deviation from hydrostatic equilibrium as expected if a shock were present. The location of the discontinuity coincides with the location of the relic, and also agrees with the location found by visual inspection of the Chandra image. The modeling suggests that the underlying density jump must be rather low $(C<2.0, \mathcal{M}<1.7)$. Some caution is required in interpreting the derived Mach number and upper limits, since it depends on the assumptions made in the modeling. Given the extra substructure in this region, as seen in the Chandra image, some of the assumptions might be incorrect. The unknown projection effects and shock geometry typically result in underestimation of the Mach number and our derived values should therefore be considered as lower limits.

Another way to constrain the Mach number, and rule out the presence of a cold front, is to determine the temperatures on both sides of the discontinuity (in a small enough region not be affected by other cluster substructure). An advantage of this method is that temperature measurements are less affected by the unknown geometry. However, the count rate in the region south of the discontinuity is very low, and therefore we are not able to obtain useful constraints on the pre-shock $\mathrm{X}$-ray gas temperature.

\section{SOAR spectroscopy and data reduction.}

The optical spectrum of the AGN host galaxy that "fuels" the relic (RA $08^{\mathrm{h}} 42^{\mathrm{m}} 13^{\mathrm{s}} .73$; DEC $-17^{\circ} 31^{\prime} 12^{\prime \prime} .1$ ) was acquired using the Goodman Spectrograph on the Southern Astrophysical Research Telescope (SOAR) telescope. The observations were conducted on November 19, 2015, as part of program SO2015B-020. The basic setup included the $6001 \mathrm{~mm}^{-1}$ grating (blue setting), and a 1 ".03 slit. The wavelength range of the observed spectrum was $3500-5500 \AA$, with a resolving power of $R \sim 1500$ and $\mathrm{S} / \mathrm{N} \sim 10 \mathrm{pixel}^{-1}$ at $4500 \AA$. Calibration frames included $\mathrm{Cu}$ and $\mathrm{HgAr}$ arc-lamp exposures, quartz-lamp flat-fields, and bias frames. The data reduction, including spectral extraction and wavelength calibration, were performed using standard IRAF routines (http://iraf.noao.edu).

A SOAR optical spectrum of the AGN host galaxy is shown in the SI. From the spectrum we determine a redshift of $z=0.164 \pm 0.001$, consistent with the galaxy being a cluster member.

\section{Subaru and Keck observations.}

We carried out deep imaging observations of the Abell 3411-3412 system with Subaru SuprimeCam [41] on 2014 February 24 in $g, r$ and $i$ with integrations of $720 \mathrm{~s}, 2880 \mathrm{~s}$, and $720 \mathrm{~s}$, respectively (P.I. D. Wittman). We took 4 exposures for $g$ and $i$, and 8 exposures for $r$. We rotated the field between each exposure $\left(30^{\circ}\right.$ for $g$ and $i$, and $15^{\circ}$ for $r$ ) in order to distribute the bleeding trails and diffraction spikes from bright stars azimuthally and later removed them by median-stacking exposures. This scheme enables us to maximize the number of detected galaxies, especially considering the number of stars at A3411-3412's low galactic latitude $\left(+15^{\circ}\right)$. The average seeing for the images was $\sim 0 .{ }^{\prime \prime} 85$. The details of the Subaru data reduction are similar to those presented in [42]. 
The primary objective for the spectroscopic survey was to maximize the number of cluster member spectroscopic redshifts. Since the SuprimeCam imaging was unavailable at the time of the spectroscopic survey planning, we took images with the Isaac Newton Telescope Wide Field Camera (INT/WFC), on 2013 October 31 in $g$ and $i$ bands, to determine the approximate red sequence of the cluster and preferentially selected those galaxies followed by potential blue cloud galaxies. These INT/WFC data were reduced with our in-house PYTHON-based pipeline [43, 44] Briefly, the sky flats for each filter were median-combined to obtain a 'master-flat'. A 'master-bias' was obtained by median-combining biases. The individual exposures were then bias-subtracted and skyflattened. Astrometric solutions were obtained by using SCAMP [45], and images were zero-point calibrated with the fourth United States Naval Observatory (USNO) CCD Astrograph Catalog (UCAC4) [46], before being stacked/combined using SWARP [47]. The difficulty of star-galaxy separation is compounded by the $1.5^{\prime \prime}-2^{\prime \prime}$ seeing of the INT/WFC imaging, which results in many blended pairs of stars passing morphological cuts designed to eliminate point sources.

We observed the Abell 3411-3412 system with the DEIMOS [48] instrument on the Keck II $10 \mathrm{~m}$ telescope on 2013, December 3 and 4. Observations were taken using $1^{\prime \prime}$ wide slits with the 1200 line $\mathrm{mm}^{-1}$ grating, resulting in a pixel scale of $0.33 \AA$ pixel $^{-1}$ and a resolution of $\sim 1 \AA$ $\left(50 \mathrm{~km} \mathrm{~s}^{-1}\right)$. The grating was tilted to a central wavelength of $6650 \AA$, resulting in a typical wavelength coverage of $5350 \AA$ to $7950 \AA$, which encompasses the spectral features $\mathrm{H} \beta$, [O 3] 4960 \& 5008, Mg 1 (b), Fe 1, Na 1 (D), [O 1], H $\alpha$, and the [N 2] doublet for galaxies near the cluster redshift. The actual wavelength coverage may be shifted by $\sim \pm 410 \AA$ depending on where the slit is located along the width of the slitmask. We observed a total of four slit masks with approximately 120 slits per mask. For each mask we took three 900 s exposures. The data reduction followed the same procedure outlined in detail in $[44,49]$.

We obtained 484 spectra with DEIMOS. Of these, we obtained reliable redshifts for 447 objects (92\%), leaving 37 spectra that were either too noisy, had ambiguous redshift solutions (e.g., those with a single emission line), or failed to reduce properly. Of the 447 reliable redshifts, 221 (49\%) are stars, indicating the difficulty of star/galaxy discrimination with low-resolution imaging at low Galactic latitude. Adopting the quality rating system of [50], in which only galaxies with secure redshifts $(Q>3)$ are considered high-quality, the removal of the stars results in 226 high quality DEIMOS galaxy spectra. Of these, 174 (77\%) fall within $0.148 \leq z \leq 0.176$ (see the SI, which is $z_{\text {cluster }} \pm 3 \times \sigma$, where $z_{\text {cluster }}=0.162$ and $\sigma$ is the approximate velocity dispersion $\left(1000 \mathrm{~km} \mathrm{~s}^{-1}\right)$. The remaining 52 high-quality spectra consist of one foreground and 51 background galaxies, comprising $0.4 \%$ and $23 \%$ of the high-quality spectra respectively. The subcluster identification and the dynamical modeling are described in the SI.

Data Availability Statement The data that support the plots within this paper and other findings of this study are available from the corresponding author upon reasonable request. 


\section{References}

[31] McMullin, J. P., Waters, B., Schiebel, D., Young, W. , Golap, K. CASA Architecture and Applications. Astronomical Data Analysis Software and Systems XVI, R. A. Shaw, F. Hill, D. J. Bell eds. Astronomical Society of the Pacific Conference Series 376, 127 (2007).

[32] Cornwell, T. J., Golap, K., Bhatnagar, S. The Noncoplanar Baselines Effect in Radio Interferometry: The W-Projection Algorithm. IEEE J. Sel. Top. Signal Process. 2, 647-657 (2008).

[33] Cornwell, T. J., Golap, K., Bhatnagar, S. W Projection: A New Algorithm for Wide Field Imaging with Radio Synthesis Arrays. Astronomical Data Analysis Software and Systems XIV P. Shopbell, M. Britton, R. Ebert, eds. Astronomical Society of the Pacific Conference Series 347, 86 (2005).

[34] van Weeren, R. J., Ogrean, G. A., Jones, C., Forman, W. R., Andrade-Santos, F., Bonafede, A., Brüggen, M., Bulbul, E., Clarke, T. E., Churazov, E., David, L., Dawson, W. A., Donahue, M., Goulding, A., Kraft, R. P., Mason, B., Merten, J., Mroczkowski, T., Murray, S. S., Nulsen, P. E. J. and Rosati, P., Roediger, E., Randall, S. W., Sayers, J., Umetsu, K., Vikhlinin, A., Zitrin, A. The Discovery of Lensed Radio and X-Ray Sources behind the Frontier Fields Cluster MACS J0717.5+3745 with the JVLA and Chandra. Astrophys. J. 817, 98 (2016).

[35] Offringa, A. R., de Bruyn, A. G., Biehl, M., Zaroubi, S., Bernardi, G., Pandey, V. N. Postcorrelation radio frequency interference classification methods Mon. Not. R. Astron. Soc. 405, 155-167 (2010).

[36] Taylor, A. R., Stil, J. M., Sunstrum, C. A Rotation Measure Image of the Sky. Astrophys. J. 702, 1230-1236 (2009).

[37] Fanaroff, B. L. and Riley, J. M. The morphology of extragalactic radio sources of high and low luminosity. Mon. Not. R. Astron. Soc. 167, 31-36 (1974).

[38] Vikhlinin, A., Markevitch, M., Murray, S. S., Jones, C., Forman, W., Van Speybroeck, L. Chandra Temperature Profiles for a Sample of Nearby Relaxed Galaxy Clusters. Astrophys. J. 628, 655-672 (2005).

[39] Eckert, D., Molendi, S., Paltani, S. The cool-core bias in X-ray galaxy cluster samples. I. Method and application to HIFLUGCS. Astron. Astrophys. 526, 79 (2011).

[40] Ogrean, G. A., Brüggen, M., van Weeren, R. J., Röttgering, H., Croston, J. H., Hoeft, M. Challenges to our understanding of radio relics: X-ray observations of the Toothbrush cluster. Mon. Not. R. Astron. Soc. 433, 812-824 (2013).

[41] Miyazaki, S., Komiyama, Y., Sekiguchi, M., Okamura, S., Doi, M., Furusawa, H., Hamabe, M., Imi, K., Kimura, M., Nakata, F., Okada, N., Ouchi, M., Shimasaku, K., Yagi, M. , Yasuda, N. Subaru Prime Focus Camera - Suprime-Cam. Publ. Astron. Soc. Jpn. 54, 833-853 (2002). 
[42] Jee, M. J., Stroe, A., Dawson, W., Wittman, D., Hoekstra, H., Brüggen, M., Röttgering, H., Sobral, D., van Weeren, R. J. MC ${ }^{2}$ : Constraining the Dark Matter Distribution of the Violent Merging Galaxy Cluster CIZA J2242.8+5301 by Piercing through the Milky Way. Astrophys. J. 802, 46 (2015).

[43] Stroe, A., Sobral, D., Röttgering, H. J. A., van Weeren, R. J. The role of cluster mergers and travelling shocks in shaping the $\mathrm{H} \alpha$ luminosity function at $z \sim 0.2$ : 'sausage' and 'toothbrush' clusters. Mon. Not. R. Astron. Soc. 438, 1377-1390 (2014).

[44] Sobral, D., Stroe, A., Dawson, W. A., Wittman, D., Jee, M. J., Röttgering, H., van Weeren, R. J., Brüggen, M. MC ${ }^{2}$ : boosted AGN and star formation activity in CIZA J2242.8+5301, a massive post-merger cluster at $\mathrm{z}=0.19$. Mon. Not. R. Astron. Soc. 450, 630-645 (2015).

[45] Bertin, E., Astronomical Data Analysis Software and Systems XV, C. Gabriel, C. Arviset, D. Ponz, S. Enrique, eds., Astronomical Society of the Pacific Conference Series 351, 112 (2006).

[46] Zacharias, N., Finch, C. T., Girard, T. M., Henden, A., Bartlett, J. L., Monet, D. G., Zacharias, M. I., Astron. J. 145, 44 (2013).

[47] Bertin, E., Mellier, Y., Radovich, M., Missonnier, G., Didelon, P., Morin, B. The TERAPIX Pipeline. Astronomical Data Analysis Software and Systems XI, D. A. Bohlender, D. Durand, T. H. Handley, eds., Astronomical Society of the Pacific Conference Series 281, 228 (2002).

[48] Faber, S. M., Phillips, A. C., Kibrick, R. I., Alcott, B., Allen, S. L., Burrous, J., Cantrall, T., Clarke, D., Coil, A. L., Cowley, D. J., Davis, M., Deich, W. T. S., Dietsch, K., Gilmore, D. K., Harper, C. A., Hilyard, D. F., Lewis, J. P., McVeigh, M., Newman, J., Osborne, J., Schiavon, R., Stover, R. J., Tucker, D., Wallace, V., Wei, M., Wirth, G., Wright, C. A. The DEIMOS spectrograph for the Keck II Telescope: integration and testing. Instrument Design and Performance for Optical/Infrared Ground-based Telescopes, M. Iye, A. F. M. Moorwood, eds. (2003), Proc. SPIE Int. Soc. Opt. Eng. 4841, 1657-1669 (2003).

[49] Dawson, W. A., Jee, M. J., Stroe, A., Ng, Y. K., Golovich, N., Wittman, D., Sobral, D., Brüggen, M., Röttgering, H. J. A. and van Weeren, R. J. MC ${ }^{2}$ : Galaxy Imaging and Redshift Analysis of the Merging Cluster CIZA J2242.8+5301. Astrophys. J. 805, 143 (2015).

[50] Newman, J. A, Cooper, M. C., Davis, M., Faber, S. M., Coil, A. L., Guhathakurta, P., Koo, D. C., Phillips, A. C., Conroy, C., Dutton, A. A., Finkbeiner, D. P., Gerke, B. F., Rosario, D. J., Weiner, B. J., Willmer, C. N. A., Yan, R., Harker, J. J., Kassin, S. A., Konidaris, N. P., Lai, K., Madgwick, D. S., Noeske, K. G., Wirth, G. D., Connolly, A. J., Kaiser, N., Kirby, E. N., Lemaux, B. C., Lin, L., Lotz, J. M., Luppino, G. A., Marinoni, C., Matthews, D. J., Metevier, A., Schiavon, R. P. The DEEP2 Galaxy Redshift Survey: Design, Observations, Data Reduction, and Redshifts. Astrophys. J. Suppl. Ser. 208, 5 (2013). 


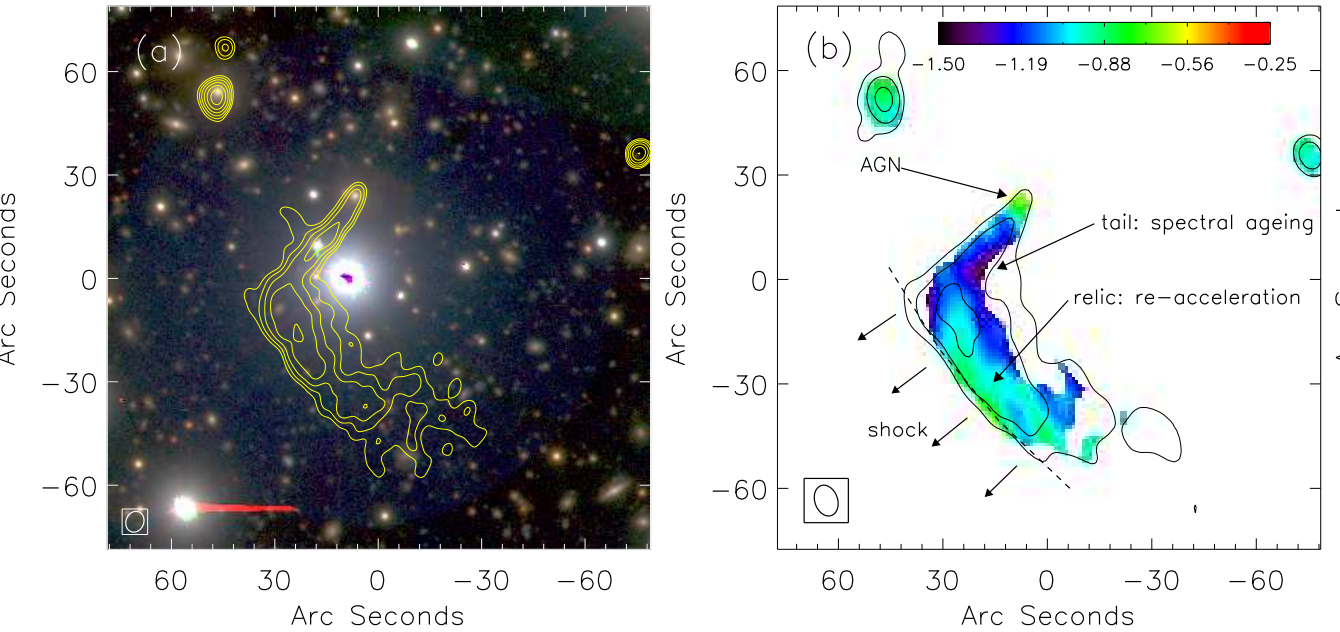


